\title{
Estimación de la salinidad en aguas freáticas del área de influencia de la desembocadura del río Sinú-Córdoba, Colombia
}

\author{
Estimating groundwater salinity in the area of the river estuary \\ Sinú-Cordoba-Colombia
}

\author{
Enrique Combatt C. ${ }^{1 *}$, Humberto Narváez M. ${ }^{1}$, Iván Darío Bustamante de la B. ${ }^{1}$
}

\section{RESUMEN}

Las sales disueltas en aguas freáticas y la proximidad de un río y costas marinas son condiciones que generan acumulación de sales en el suelo. El objetivo del trabajo fue estudiar la tendencia de la salinidad de las aguas freáticas someras en el área de influencia de la desembocadura del río Sinú en Córdoba-Colombia. El área de estudio de $82 \mathrm{~km}^{2}$ se subdividió en cuatro localidades próximas a la desembocadura del río Sinú, donde se instalaron 39 pozos de observación distribuidos en 25 pozos en vértices principales con espaciamiento de $2 \mathrm{~km}$ entre sí y 14 en vértices auxiliares con espaciamiento de $1 \mathrm{~km}$ respecto de los principales. Para el muestreo de las aguas freáticas se definieron los periodos correspondientes a la época seca y lluviosa del año. El análisis químico de las muestras de agua se realizó en el Laboratorio de Suelos y Aguas de la Universidad de Córdoba. En las muestras se determinó la conductividad eléctrica (CE), $\mathrm{pH}, \mathrm{Ca}, \mathrm{Mg}, \mathrm{Na}, \mathrm{K}, \mathrm{HCO}_{3}, \mathrm{CO}_{3}$ y $\mathrm{SO}_{4}$. Los datos obtenidos fueron analizados mediante análisis de varianza y pruebas de separación de medias (Tukey) ( $\leq 0.05$ ), además análisis de correlación simple entre CE e iones solubles y la estimación de modelos de CE por el método de regresión por pasos (The Stepwise regresión procedure) y clasificación de las aguas freáticas basándose en la contribución de cada variable en los modelos. Los resultados indican que las aguas son marcadamente salinas en localidades próximas a la desembocadura del río Sinú y asociadas a los niveles de iones $\mathrm{Cl}, \mathrm{Na}$ y $\mathrm{Mg}$. Los modelos de CE calculados para las localidades presentan $\mathrm{R}^{2} \geq 0,94$; indicando alto grado de confiabilidad para la estimación de la CE, igualmente se determinó que el tipo de aguas que predomina son las cloruradas en el periodo lluvioso y en el periodo seco no predomina ningún tipo de sal.

Palabras clave: Estuarios marinos, conductividad eléctrica, aguas freáticas, modelos de regresión, clasificación iónica de aguas.

\section{ABSTRACT}

The dissolved salts in groundwater and proximity to a river and coastal seas are conditions that generate accumulation of salts in the soil. The objective was to study the trend of the salinity of the shallow groundwater in the catchment area of the river mouth of Sinú River in Cordoba - Colombia. The study area of $82 \mathrm{~km}^{2}$ was divided into four locations near the river mouth of the Sinú River, where 39 observation wells distributed in 25 wells in main vertices with spacing of $2 \mathrm{~km}$ apart and 14 auxiliary vertices with spacing $1 \mathrm{~km}$ of the main. For the sampling of groundwater two periods were chosen and corresponded to season of dry and rainy seasons. Chemical analysis was performed in the Laboratory of Soil and Water at the University of Córdoba. In the samples were evaluated the electrical conductivity (EC), $\mathrm{pH}, \mathrm{Ca}, \mathrm{Mg}, \mathrm{Na}, \mathrm{K}, \mathrm{HCO}_{3}, \mathrm{CO}_{3}$ and $\mathrm{SO}_{4}$. The data obtained were analyzed by analysis of variance and tests separation of means (Tukey) $(p \leq 0.05)$. Simple correlation analysis between CE and soluble ions and estimating EC models by the method of regression by Steps (The Stepwise regression procedure) and water classification based on the contribution of each variable in the models. The results indicate that the water is strongly saline in locations near the river mouth of the Sinú River and associated levels to ions $\mathrm{Cl}, \mathrm{Na}$ and $\mathrm{Mg}$. The CE models calculated for localities have $R^{2} \geq 0,94$ indicating high reliability for estimating the EC. Also were found that the predominant types of waters are as chloride classified in the rainy season and the dry season not prevails any salt.

Key words: marine estuaries, electric conductivity, phreatic water, models of multiple lineal regressions, ionic classification of water.

\footnotetext{
$1 \quad$ Facultad de Ciencias Agrícolas, Universidad de Córdoba, Carrera 6 \# \# 76-103. Telefax 574 7860255. Montería, Córdoba, Colombia.

* Autor para correspondencia: ecombatt@ fca.edu.co
}

Fecha de Recepción: 16 Febrero, 2015.

Fecha de Aceptación: 8 Junio, 2015. 


\section{Introducción}

En la producción agropecuaria se pueden presentar problemas de salinidad que están marcadamente influenciados por un nivel freático poco profundo que se encuentra a menos de dos metros de la superficie del suelo. Esto es ocasionado por que las sales se acumulan en el agua freática y con frecuencia se convierten en una importante fuente adicional de sales, que se mueve hacia arriba por los procesos de capilaridad, depositándose en la zona de crecimiento de las raíces de los cultivos.

De acuerdo con Tanji (1990), la elevación del manto freático causado por el riego o debido a la proximidad de una zona agroecológica a un río y las precipitaciones pueden generar una acumulación de sales en el suelo. La acumulación de sales ocasiona problema de fitotoxicidad a los cultivos al convertirse en una barrera para el crecimiento de las raíces y restringirlas a un volumen de suelo muy pequeño, ocasionado por la alta concentración de sales y aumento de la presión osmótica (Negrete et al., 2011). Bustamante (1993) indica que la falta de un adecuado drenaje subsuperficial y la presencia de una napa freática somera agravan los problemas de salinidad y complican su manejo.

La característica del agua freática está determinada no solo por la cantidad de aniones y cationes presente, sino también por el tipo de sales que se pueden formar. Por esto el estudio de la composición iónica del agua es de gran importancia en la agricultura, debido a los efectos adversos que provocan las sales sobre el suelo y los cultivos. Es así que las comparaciones de las composiciones de los iones disueltos en el agua freática guardan siempre una cierta relación con la composición de los iones en los suelos, y dependiendo de las sales que se forman se pueden presentar problemas por sequía osmótica, toxicidad debida a la excesiva absorción de cloro y sodio y desbalance nutricional (Rodríguez-Pérez (2006; Cortés-Jiménez et al., 2009).

Para el estudio de las aguas existen diversas formas de clasificación y la mayoría de estas se basan en la relación de absorción de sodio (RAS) y la conductividad eléctrica (CE) (Shalhevet y Kamburov, 1976; Porta et al., 1999; Silva, 2000). El estudio de la CE es el método más empleado para expresar la concentración de las sales solubles en agua, la que se refiere al inverso de la resistividad eléctrica, que corresponde a la capacidad del agua para conducir electricidad, que crece proporcionalmente a medida que la concentración de sales aumenta (Maia et al., 2001; Holanda y Amorim, 1997; Ferreira, 1997).

Las sales están presentes en el agua en cantidades relativamente pequeñas, y su concentración depende de la actividad iónica de los iones en solución y de la solubilidad de sus compuestos. Porta et al., (1999) exponen que la solubilidad de las sales es una propiedad muy importante, porque además de afectar su movilidad y precipitación regula su máxima concentración en la solución del suelo.

En zonas cercanas al estuario del río Sinú se presentan bajos rendimientos en los cultivos tradicionales dependiendo de la época del año, lo que está incidiendo en la reducción del área cultivable. El problema es asociado principalmente al incremento de sales en los primeros centímetros de profundidad del perfil del suelo. Por lo anterior, la salinización de áreas cultivables en zonas adyacentes a la desembocadura del río Sinú, amerita una evaluación puntual que permita vislumbrar la magnitud del fenómeno, debido a las implicaciones socioeconómicas que esta representa al disminuir la producción agrícola y la rentabilidad de los cultivos. Por ello, el objetivo de la presente investigación fue generar modelos de regresión lineal múltiple para la estimación de la salinidad en aguas freáticas en periodos secos y lluviosos en área de influencia de la desembocadura del río Sinú.

\section{Materiales y Métodos}

Para la presente investigación fueron utilizados datos provenientes de la Fase II-VI del proyecto titulado "Diagnóstico general del nivel de salinización en la zona aledaña a la desembocadura del río Sinú y su impacto socioeconómico", Período Abril/2002noviembre/2007. La zona de estudio corresponde a $82 \mathrm{~km}^{2}$ del área de influencia de la desembocadura del río Sinú, concentrada en los últimos $22 \mathrm{~km}$ de su recorrido y cuyos puntos extremos tienen coordenadas geográficas $9^{\circ} 27^{\prime} 22,3$ " de latitud N; $75^{\circ} 56^{\prime} 34,5^{\prime \prime}$ de longitud W y $9^{\circ} 19^{\prime} 34,6$ " de latitud $\mathrm{N} ; 75^{\circ} 51^{\prime}$ '4,5" de longitud W.

Esta zona limita al norte con el océano Atlántico, al sur con los corregimientos La Doctrina y Las Cañas, al este con las Ciénagas de Ostional y al oeste por el complejo de lomeríos de José Manuel y Calle Ralita, en comprensión territorial de los municipios de San Bernardo del Viento y Lórica, del departamento de Córdoba, Colombia, y de acuerdo con la clasificación de Holdridge, esta área 
tiene como zona de vida, la de bosque seco tropical (bs-T) con regímenes de precipitaciones promedio de $1.425 \mathrm{~mm}$ año ${ }^{-1}$, temperatura media de $27,6{ }^{\circ} \mathrm{C}$ y humedad relativa del $84 \%$ (Palencia et al., 2006).

La zona de estudio se subdividió en cuatro localidades L1, L2, L3 y L4, basándose en la proximidad de los pozos muestreados (Figura 1). El muestreo comprendió 39 pozos de observación distribuidos en 25 pozos en vértices principales con espaciamiento de $2 \mathrm{~km}$ entre sí y 14 en vértices auxiliares con espaciamiento de $1 \mathrm{~km}$ respecto de los principales. Para el estudio se tomaron 18 muestreos de aguas freáticas correspondientes al periodo mayo/2002-enero/2003 y lecturas en julio 2003, Mayo 2004; enero, abril y noviembre de 2005, mayo y noviembre de 2006; abril y noviembre del 2007.

Para la zona de estudio se definieron los periodos correspondientes a la época seca y lluviosa del año. Para ello se analizó el registro histórico de precipitación de 16 años (1990 a 2005) de la Estación Meteorológica del municipio de San Bernardo Viento. Como época lluviosa se consideraron los meses que presentaron más del $10 \%$ de la precipitación media anual, que abarca el periodo de mayo a noviembre (más del 92\% de la lluvia total) y por lo tanto, la época seca correspondió al periodo de diciembre a abril.

Las muestras de aguas freáticas fueron procesadas por el Laboratorio de Suelos y Aguas de la Universidad de Córdoba, según normas de Apha (1995) y se determinaron las siguientes características: conductividad eléctrica $(\mathrm{CE})$ por método conductimétrico, cationes $\mathrm{Ca}^{2+}, \mathrm{Mg}^{2+}$ por espectrofotometría de absorción atómica $\mathrm{Na}^{+}$y $\mathrm{K}^{+}$por espectrofotometría de emisión atómica, aniones $\mathrm{Cl}^{-}$por titulación volumétrica con nitrato de plata $0,0141 \mathrm{~N}, \mathrm{HCO}_{3}^{-}$y $\mathrm{CO}_{3}{ }^{2-}$ por titulación volumétrica con $\mathrm{H}_{2} \mathrm{SO}_{4}=0,02 \mathrm{~N} \mathrm{y} \mathrm{SO}_{4}{ }^{=}$por el método turbidimétrico. La relación de absorción de sodio (RAS) fue determinada según la siguiente ecuación: $\mathrm{RAS}=\mathrm{Na} /((\mathrm{Ca}+\mathrm{Mg}) / 2)^{1 / 2}$

Los datos de CE, cationes y aniones entre localidades en periodos secos y lluviosos fueron sometidos a análisis de varianza y pruebas de separación de medias de Tukey ( $\mathrm{p} \leq 0,05)$, así como análisis de correlación simple (Pearson) entre $\mathrm{CE}$ e iones determinados en aguas freáticas. Se

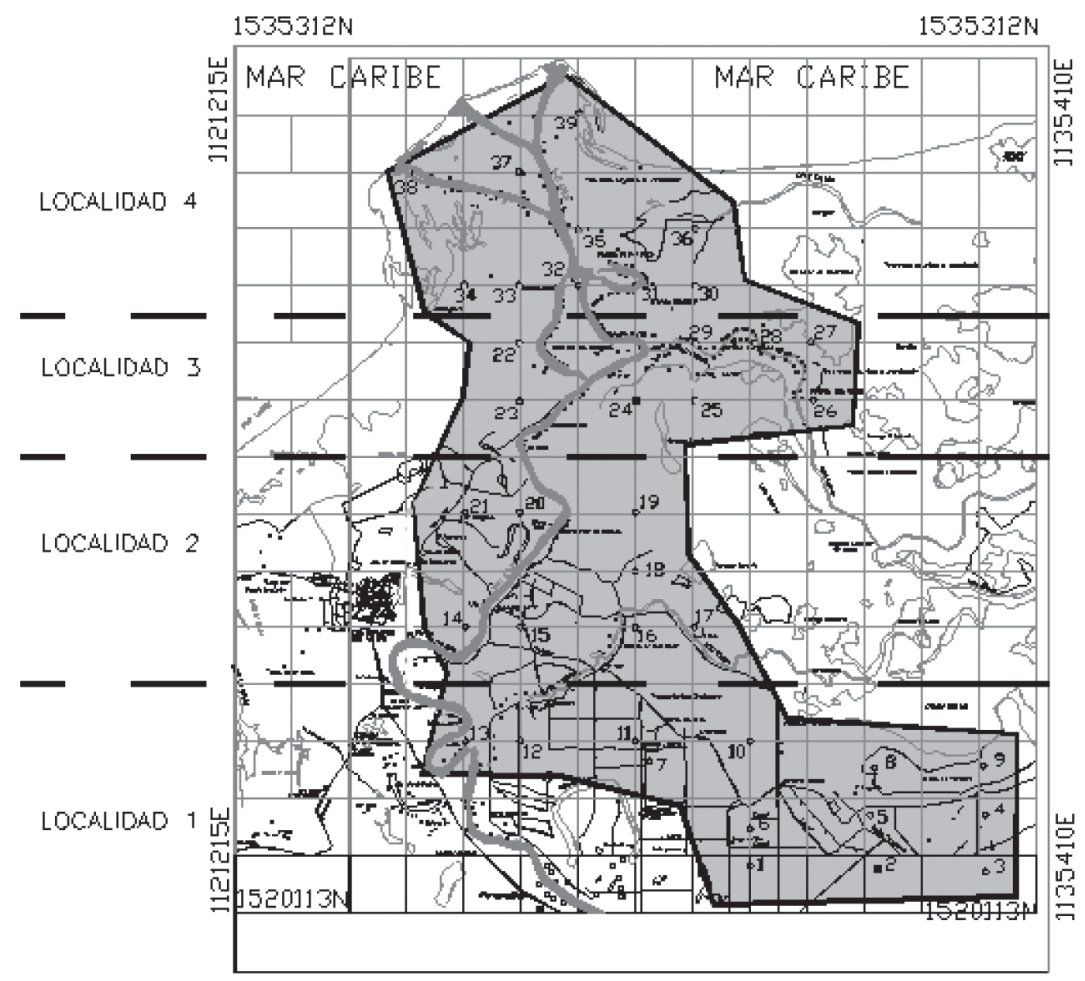

Figura 1. Localidades de la zona de estudio en los últimos $22 \mathrm{~km}$ del río Sinú en Córdoba, Colombia. 
estimaron modelos de regresión lineal múltiple para la zona de estudio en periodos secos y lluviosos por localidad y a nivel general se consideraron todas las localidades por periodo, donde los iones $\mathrm{Ca}, \mathrm{Mg}$, $\mathrm{Na}, \mathrm{Cl}, \mathrm{K}, \mathrm{HCO}_{3}, \mathrm{CO}_{3}, \mathrm{y} \mathrm{SO}_{4}$ fueron las variables independientes y la $\mathrm{CE}$ la variable dependiente. La ecuación de regresión tuvo la siguiente forma:

$$
Y=a+b X_{i}+c X_{i+1}+d X_{i+2}+d X_{i+n}+e_{i}
$$

Donde,

$Y$ : conductividad eléctrica o variable dependiente, $\mathrm{dS} \mathrm{m}^{-1}$

$X i$ : variable independiente

$a$ : constante de regresión

$b, c, d$ : coeficientes angulares de la variable, y

$e$ : error aleatorio

El análisis de los datos se realizó utilizando el programa estadístico SAS (Statistical Analysis System) v. 9.2, siendo obtenidos los modelos por el método de regresión por pasos (The Stepwise regresion procedure). Para la clasificación de las aguas freáticas de acuerdo con su composición iónica como sódica, cálcica, magnésica y clorurada se utilizó la metodología planteada por Maia et al. (2001), donde se consideran las variables independientes que integran los modelos de regresión múltiple y su contribución a los modelos. Esta contribución cuantitativa se estima por el aumento observado en la suma de cuadrados debido a la regresión, cuando se adicionan variables al modelo. Sumándose todos los pesos de las variables iguales al $100 \%$, se realizó una proporción porcentual de cada variable. El mayor peso, multiplicado por 0,9 es el valor límite para que los iones entren en la clasificación del agua.

\section{Resultados y Discusión}

Se obtuvieron modelos de regresión lineal múltiple para la $\mathrm{CE}$ con coeficientes de determinación $\left(R^{2}\right)$ entre 0,94 y 0,98 en las diferentes localidades para el periodo lluvioso y entre 0,94 y 0,98 en el periodo seco, indicando el alto grado de confiabilidad que presentan estas ecuaciones para estimar los valores de $\mathrm{CE}$ en función de la concentración de los iones que integran los modelos. Los modelos generados presentan entre dos y tres parámetros significativos y altamente significativos, donde la contribución del ion $\mathrm{Cl}^{-}$fue predominante en todas las localidades en el periodo lluvioso, mientras que en el periodo seco la contribución predominante fue diferencial a nivel de localidades (Tabla 1).

Se presentaron algunos coeficientes negativos en los parámetros de los modelos y esta condición obedece posiblemente a factores como la solubilidad de los iones, su radio de hidratación, concentración de $\mathrm{CO}_{2}$ y la disponibilidad del agua y su movimiento, que influyen en la formación de los compuestos de variada solubilidad que intervienen en la $\mathrm{CE}$ de las aguas freáticas. En estas condiciones se presenta formación de compuestos que pueden precipitar

Tabla 1. Ecuaciones de regresión lineal múltiple, coeficiente de determinación y contribución de las variables independientes para la estimación de la CE en aguas freáticas en el área de influencia de la desembocadura del río Sinú en periodos lluviosos y secos.

\begin{tabular}{|c|c|c|}
\hline \multicolumn{3}{|c|}{ Período lluvioso: mayo-noviembre } \\
\hline Localidad & Ecuación de regresión lineal múltiple & Contribución \\
\hline L1 & $\mathrm{CE}=0,47787+0,10322 \mathrm{Cl} * * *+0,15262 \mathrm{CO}_{3} * * *$ & $\mathrm{Cl}>\mathrm{CO}_{3}$ \\
\hline L2 & $\mathrm{CE}=1,16749-0,01948 \mathrm{Mg}^{* *}+0,09862 \mathrm{Cl}^{* * *}$ & $\mathrm{Cl}>\mathrm{Mg}$ \\
\hline L3 & $\mathrm{CE}=0,53232+0,05459 \mathrm{Mg} * * *+0,08123 \mathrm{Cl}^{* * *}+0,52369 \mathrm{CO}_{3}^{* *}$ & $\mathrm{Cl}>\mathrm{Mg}>\mathrm{CO}_{3}$ \\
\hline L4 & $\mathrm{CE}=0,86620+0,14545 \mathrm{Ca}^{*}+0,09382 \mathrm{Cl} * * *$ & $\mathrm{Cl}>\mathrm{Ca}$ \\
\hline General $^{+}$ & $\mathrm{CE}=0,80431-0,01074 \mathrm{Na}^{* * *}+0,10503 \mathrm{Cl}^{* * *}+0,21753 \mathrm{CO}_{3} * * *$ & $\mathrm{Cl}>\mathrm{CO}_{3}>\mathrm{Na}$ \\
\hline \multicolumn{3}{|c|}{ Período seco: diciembre-abril } \\
\hline L1 & $\mathrm{CE}=0,13884+0,16623 \mathrm{Ca}^{* * *}+0,09818 \mathrm{Cl}^{* * *}+0.13911 \mathrm{CO}_{3} * * *$ & $\mathrm{Cl}>\mathrm{Ca}>\mathrm{CO}_{3}$ \\
\hline $\mathrm{L} 2$ & $\mathrm{CE}=-0,44153+0,25202 \mathrm{Ca}^{* *}+0,14892 \mathrm{Mg}^{* * *}+0,07231 \mathrm{Na}^{* * * *}$ & $\mathrm{Na}>\mathrm{Mg}>\mathrm{Ca}$ \\
\hline L3 & $\mathrm{CE}=-1,25260+0,31070 \mathrm{Mg} * * *+7,99499 \mathrm{~K}^{* * *}$ & $\mathrm{Mg}>\mathrm{K}$ \\
\hline $\mathrm{L} 4$ & $\mathrm{CE}=1,19649+0,24770 \mathrm{Mg}^{* * *}+5,62110 \mathrm{~K} * * *-0,58267 \mathrm{SO}_{4} * * *$ & $\mathrm{~K}>\mathrm{Mg}>\mathrm{SO}_{4}$ \\
\hline General $^{+}$ & $\mathrm{CE}=-0,64890+0,30485 \mathrm{Mg}^{* * *}+1,46486 \mathrm{~K}^{* * *}+0,93463 \mathrm{CO}_{3}^{4 * * *}$ & $\mathrm{Mg}>\mathrm{K}>\mathrm{CO}_{3}^{4}$ \\
\hline
\end{tabular}

$\mathrm{CE}=$ Conductividad eléctrica en dS $\cdot \mathrm{m}^{-1} \mathrm{Ca}, \mathrm{Mg}, \mathrm{Na}, \mathrm{K}, \mathrm{Cl}, \mathrm{CO}_{3}, \mathrm{SO}_{4}$ en $\mathrm{cmol} \mathrm{L}^{-1} \mathrm{R}^{2}$ : coeficiente de determinación. Se consideran todas las localidades, *significativo (10\%), **significativo (5\%), ***altamente significativo (1\%). 
cuando la concentración de ácido carbónico aumenta y si el ácido carbónico aumenta puede incrementar la solubilidad de fuentes de carbonatos, como piedras calizas y otros minerales.

Según Coto et al. (2012), la presión parcial de $\mathrm{CO}_{2}$ tiene un efecto marcado, ya que existe una directa relación con la solución de $\mathrm{pH}$, y este puede modificar la cantidad de especies iónicas presentes en la solución acuosa, y por lo tanto, el aumento de la solubilidad del carbonato de calcio

Con la evaluación de los modelos se identificaron diferentes tipos de agua en cuanto a su composición iónica. Para el periodo lluvioso las aguas fueron clasificadas como cloruradas en todas las localidades y mediante el modelo general también se estimaron aguas cloruradas, mientras que para el periodo seco en las localidades L1, L2, L3 y L4 sus aguas freáticas presentaron diferentes clasificaciones entre ellas, aguas cloruradas, sódicas, magnésicas y potásicas respectivamente y como aguas magnésicas para el modelo general (Tabla 2). Los modelos presentaron $\mathrm{R}^{2}$ próximos a los obtenidos para aguas por Maia et al. (2001), los que estuvieron entre 0,96 y 0,99.

En cuanto a los contenidos de los iones en las aguas freáticas, el análisis estadístico detectó diferencias estadísticas significativas $(\operatorname{Pr}<0.05)$ entre localidades para la $\mathrm{CE}$ e iones $\mathrm{Ca}, \mathrm{Mg}, \mathrm{Na}$, $\mathrm{Cl}, \mathrm{SO}_{4}$ y RAS en el periodo lluvioso y $\mathrm{CE}, \mathrm{Ca}$, $\mathrm{Mg}, \mathrm{Na}, \mathrm{K}, \mathrm{Cl}, \mathrm{SO}_{4}$, y RAS en el periodo seco (Tabla 3). En los dos periodos la localidad 1 (L1) presentó los niveles estadísticamente más bajos de $\mathrm{CE}$, mientras que las localidades 2, 3 y 4 (L2, L3 y L4), la CE presenta una relación directa con la proximidad a la zona costera.

De acuerdo con la clasificación de la calidad del agua propuesta por la FAO (Ayers y Westcot, 1985) en los dos periodos la localidad más retirada de la zona costanera (L1) presentó aguas con grado de restricción de salinidad moderada ( $\mathrm{CE}=0,7 \mathrm{a}$ 3,0 dS m$~^{-1}$ ), mientras en las L2, L3 y L4, las aguas presentaron una restricción severa por salinidad $\left(\mathrm{CE}>3,0 \mathrm{dS} \mathrm{m}^{-1}\right)$, de igual forma, basándose en esta misma clasificación a nivel general, evaluando localidades y periodos no se presentó ninguna restricción de estas aguas para problemas potenciales de infiltración debido a la salinidad de estas aguas (Tabla 4).

De acuerdo con los niveles reportados para aguas agrícolas por Ayers y Westcot (1985), en la evaluación de los periodos los iones $\mathrm{Cl}$ y $\mathrm{Na}$ presentaron valores en concentraciones ubicados
Tabla 2. Peso en la suma de cuadrados (S.C) y en porcentaje (\%) de las variables de cada modelo para la clasificación de las aguas freáticas.

\begin{tabular}{|c|c|c|c|c|}
\hline \multicolumn{5}{|c|}{ Período lluvioso: mayo-noviembre } \\
\hline Localidad & Ion & Peso S.C & Peso $(\%)$ & Clasificación \\
\hline \multirow[t]{2}{*}{ L1 } & $\mathrm{Cl}$ & 2061,01 & 99,64 & Clorurada \\
\hline & $\mathrm{CO}_{3}$ & 7,42 & 0,36 & \\
\hline \multirow[t]{2}{*}{$\mathrm{L} 2$} & $\mathrm{Cl}$ & 1548,79 & 99,39 & Clorurada \\
\hline & $\mathrm{Mg}$ & 9,51 & 0,64 & \\
\hline \multirow[t]{3}{*}{ L3 } & $\mathrm{Cl}$ & 1817,94 & 96,21 & Clorurada \\
\hline & $\mathrm{Mg}$ & 72,46 & 2,26 & \\
\hline & $\mathrm{CO}_{3}$ & 28,83 & 1,53 & \\
\hline \multirow[t]{2}{*}{ L4 } & $\mathrm{Cl}^{3}$ & 722,08 & 99,44 & Clorurada \\
\hline & $\mathrm{Ca}$ & 4,08 & 0,56 & \\
\hline \multirow[t]{3}{*}{ General } & $\mathrm{Cl}$ & 2020,15 & 97,23 & Clorurada \\
\hline & $\mathrm{CO}_{3}$ & 34,73 & 1,67 & \\
\hline & $\mathrm{Na}$ & 22,71 & 1,10 & \\
\hline \multicolumn{5}{|c|}{ Período seco: diciembre-abril } \\
\hline \multirow[t]{3}{*}{ L1 } & $\mathrm{Cl}$ & 28,85 & 94,84 & Clorurada \\
\hline & $\mathrm{Ca}$ & 1,06 & 3,48 & \\
\hline & $\mathrm{CO}_{3}$ & 0,51 & 14,68 & \\
\hline \multirow[t]{3}{*}{ L2 } & $\mathrm{Na}$ & 185,52 & 86,39 & Sódica \\
\hline & $\mathrm{Mg}$ & 15,38 & 7,28 & \\
\hline & $\mathrm{Ca}$ & 10,37 & 6,33 & \\
\hline \multirow[t]{2}{*}{ L3 } & $\mathrm{Mg}$ & 231,08 & 55,35 & Magnésica \\
\hline & $\mathrm{K}$ & 186,42 & 44,65 & \\
\hline \multirow[t]{3}{*}{ L4 } & $\mathrm{K}$ & 1285,01 & 36,82 & Potásica \\
\hline & $\mathrm{Mg}$ & 1145,04 & 32,81 & \\
\hline & $\mathrm{SO}_{4}$ & 1060,06 & 30,37 & \\
\hline \multirow[t]{3}{*}{ General } & $\mathrm{Mg}$ & 3221,71 & 88,22 & Magnésica \\
\hline & $\mathrm{K}$ & 284,24 & 7,78 & \\
\hline & $\mathrm{CO}_{3}$ & 145,77 & 4,00 & \\
\hline
\end{tabular}

dentro de los niveles que son de 0 a 30 y de 0 a 40 $\mathrm{cmol} \cdot \mathrm{L}^{-1}$ para la localidad L1. Para el caso del ion $\mathrm{Mg}$ todos los valores fueron superiores, mientras que Ca estuvo enmarcado dentro de los niveles de aguas agrícolas.

Es importante resaltar que los iones $\mathrm{Cl}, \mathrm{Na}$ y $\mathrm{Mg}$ presentaron una tendencia muy similar a la $\mathrm{CE}$ en cuanto a localidades en los dos periodos (Tabla 4). Por ello, los valores de CE pueden estar siendo explicados por la alta concentración de los iones $\mathrm{Cl}$, Na y Mg. Esta asociación se fortalece con los coeficientes de correlación obtenidos, los cuales alcanzaron valores de 0,$99 ; 0,96$ y 0,81 para $\mathrm{Cl}, \mathrm{Na}$ y $\mathrm{Mg}$ respectivamente en la época lluviosa y del orden de 0,$96 ; 0,94$ y 0,90 para estos mismos iones en la época seca. De igual forma, iones como $\mathrm{CO}_{3} \mathrm{y} \mathrm{SO}_{4}$ que aparecen aportando en la generación de los modelos de CE, presentaron correlaciones máximas de 0,44 y 0,59 respectivamente (Tabla 5). Esto indica que las concentraciones per-se de los 
Tabla 3. Cuadrados medios y niveles de significancia de CE, iones y RAS en aguas freáticas para diferentes localidades de la desembocadura del río Sinú en periodos lluviosos y secos.

Período lluvioso: mayo-noviembre

\begin{tabular}{|c|c|c|c|c|c|c|c|c|c|c|}
\hline $\begin{array}{l}\text { F. V. } \\
\text { Localidades }\end{array}$ & $\begin{array}{c}\mathrm{CE} \\
187,8^{* *}\end{array}$ & $\begin{array}{c}\mathrm{Ca} \\
296,5^{* *}\end{array}$ & $\begin{array}{c}\mathrm{Mg} \\
1981,6^{* *}\end{array}$ & $\begin{array}{c}\mathrm{Na} \\
10295,8 * *\end{array}$ & $\begin{array}{c}\mathrm{K} \\
124,1 \mathrm{~ns}\end{array}$ & $\begin{array}{c}\mathrm{Cl} \\
21971,2 * *\end{array}$ & $\begin{array}{l}\mathrm{HCO}_{3} \\
3,2 \mathrm{~ns}\end{array}$ & $\begin{array}{c}\mathrm{CO}_{3} \\
3,9 \mathrm{~ns}\end{array}$ & $\begin{array}{c}\mathrm{SO}_{4} \\
1158 *\end{array}$ & $\begin{array}{l}\text { RAS } \\
320^{* *}\end{array}$ \\
\hline $\mathrm{CV}$ & 32,0 & 41,6 & 72,9 & 55,2 & 151,3 & 47,8 & 20,5 & 81,7 & 100,4 & 54,7 \\
\hline $\mathrm{R}^{2}$ & 0,81 & 0,69 & 0,55 & 0,68 & 0,79 & 0,69 & 0,72 & 0,45 & 0,55 & 0,62 \\
\hline \multicolumn{11}{|c|}{ Período seco: diciembre -abril } \\
\hline F. V. & $\mathrm{CE}$ & $\mathrm{Ca}$ & $\mathrm{Mg}$ & $\mathrm{Na}$ & K & $\mathrm{Cl}$ & $\mathrm{HCO}_{3}$ & $\mathrm{CO}_{3}$ & $\mathrm{SO}_{4}$ & RAS \\
\hline Localidades & $93,37 * *$ & $190,85^{* *}$ & $586,75^{* *}$ & $5797,22 * *$ & $1,87 * *$ & $11443,71 *$ & $0,07 \mathrm{~ns}$ & $0,40 \mathrm{~ns}$ & $68,48^{*}$ & $163,17 * *$ \\
\hline $\mathrm{CV}$ & 30,8 & 34,5 & 33,3 & 45,5 & 56,9 & 44,0 & 13,9 & 29,1 & 63,1 & 35,8 \\
\hline $\mathrm{R}^{2}$ & 0,86 & 0,82 & 0,79 & 0,76 & 0,78 & 0,78 & 0,82 & 0,84 & 0,76 & 0,73 \\
\hline
\end{tabular}

*Efectos significativos (5\%), ** efectos altamente significativos $(\leq 1 \%)$. CE: Conductividad eléctrica, RAS: Relación de adsorción de sodio, F.V.: fuente de variación; CV: coeficiente de variación; $\mathrm{R}^{2}$ : coeficiente de determinación.

Tabla 4. Niveles de CE, iones y RAS en aguas freáticas para diferentes localidades de la desembocadura del río Sinú en periodos lluviosos y secos. Periodo lluvioso: mayo-noviembre.

Período lluvioso: mayo-noviembre

\begin{tabular}{|c|c|c|c|c|c|c|c|c|c|c|}
\hline \multirow{2}{*}{ Localidades } & \multirow{2}{*}{$\begin{array}{c}\mathrm{CE} \\
-\mathrm{dS} \cdot \mathrm{m}^{-1}-\end{array}$} & $\mathrm{Ca}$ & $\mathrm{Mg}$ & $\mathrm{Na}$ & $\mathrm{K}$ & $\mathrm{Cl}$ & $\mathrm{HCO}_{3}$ & $\mathrm{CO}_{3}$ & $\mathrm{SO}_{4}$ & RAS \\
\hline & & \multicolumn{8}{|c|}{$\mathrm{cmol} \mathrm{L}^{-1}$} & \\
\hline L1 & $2,96 b$ & $2,24 \mathrm{~b}$ & $6,28 b$ & $20,75 b$ & $1,65 \mathrm{a}$ & $21,43 a$ & $4,69 a$ & $3,10 \mathrm{a}$ & $4,73 b$ & $8,69 \mathrm{a}$ \\
\hline L2 & $9,79 \mathrm{a}$ & $12,28 \mathrm{a}$ & $35,36 \mathrm{a}$ & $65,09 \mathrm{a}$ & $6,49 \mathrm{a}$ & $87,39 \mathrm{a}$ & $5,22 \mathrm{a}$ & $1,81 \mathrm{a}$ & $23,21 \mathrm{ab}$ & $14,38 \mathrm{ab}$ \\
\hline L3 & $10,48 \mathrm{a}$ & $10,99 \mathrm{a}$ & $22,28 \mathrm{ab}$ & $77,58 \mathrm{a}$ & $7,81 \mathrm{a}$ & $105,7 \mathrm{a}$ & $5,79 a$ & $2,22 \mathrm{a}$ & $25,39 a$ & $19,99 \mathrm{a}$ \\
\hline L4 & $11,17 \mathrm{a}$ & $11,92 \mathrm{a}$ & $29,75 \mathrm{a}$ & $82,57 \mathrm{a}$ & $8,49 \mathrm{a}$ & $110,5 \mathrm{a}$ & $5,68 \mathrm{a}$ & $2,58 \mathrm{a}$ & $21,35 \mathrm{ab}$ & $18,02 \mathrm{a}$ \\
\hline Promedio & 8,60 & 9,36 & 23,51 & 61,50 & 6,11 & 81,26 & 5,35 & 2,42 & 18,67 & 15,27 \\
\hline \multicolumn{11}{|c|}{ Período seco: diciembre-abril } \\
\hline Localidades & $\mathrm{CE}$ & $\mathrm{Ca}$ & $\mathrm{Mg}$ & $\mathrm{Na}$ & $\mathrm{K}$ & $\mathrm{Cl}$ & $\mathrm{HCO}_{3}$ & $\mathrm{CO}_{3}$ & $\mathrm{SO}_{4}$ & RAS \\
\hline L1 & $1,66 \mathrm{~b}$ & $1,58 \mathrm{~b}$ & $5,05 \mathrm{~b}$ & $11,19 \mathrm{~b}$ & $0,15 b$ & $11,62 b$ & $4,35 \mathrm{a}$ & $1,49 a$ & $1,09 \mathrm{~b}$ & $6,12 b$ \\
\hline L2 & $8,67 \mathrm{a}$ & $14,23 \mathrm{a}$ & $27,69 \mathrm{a}$ & $66,07 \mathrm{a}$ & $0,60 \mathrm{~b}$ & $72,03 \mathrm{ab}$ & $4,61 \mathrm{a}$ & $2,15 \mathrm{a}$ & $9,37 \mathrm{a}$ & $14,37 \mathrm{ab}$ \\
\hline L3 & $10,01 \mathrm{a}$ & $14,93 a$ & $24,64 \mathrm{a}$ & $65,47 \mathrm{a}$ & $0,51 b$ & $95,01 \mathrm{a}$ & $4,38 \mathrm{a}$ & $2,00 \mathrm{a}$ & $7,92 \mathrm{ab}$ & $15,08 \mathrm{ab}$ \\
\hline $\mathrm{L} 4$ & $11,38 \mathrm{a}$ & $11,67 \mathrm{a}$ & $27,31 \mathrm{a}$ & $92,18 \mathrm{a}$ & $1,58 \mathrm{a}$ & $124,42 \mathrm{a}$ & $4,49 \mathrm{a}$ & $1,79 \mathrm{a}$ & $7,76 \mathrm{ab}$ & $19,88 \mathrm{a}$ \\
\hline Promedio & 7,93 & 10,60 & 21,17 & 58,73 & 0,71 & 75,77 & 4,46 & 1,85 & 6,53 & 13,86 \\
\hline
\end{tabular}

Promedios dentro de cada columna acompañados de letras distintas denotan diferencias significativas según la prueba de Tukey (5\%). CE: Conductividad eléctrica, RAS: Relación de adsorción de sodio.

Tabla 5. Correlación simple (Pearson) entre CE y cationes y aniones en aguas freáticas para diferentes localidades de la desembocadura del río Sinú en periodos lluviosos y secos.

\begin{tabular}{|c|c|c|c|c|c|c|c|c|c|}
\hline \multicolumn{10}{|c|}{ Período lluvioso: mayo-noviembre } \\
\hline Localidad & & $\mathrm{Ca}$ & $\mathrm{Mg}$ & $\mathrm{Na}$ & $\mathrm{K}$ & $\mathrm{Cl}$ & $\mathrm{HCO}_{3}$ & $\mathrm{CO}_{3}$ & $\mathrm{SO}_{4}$ \\
\hline $\mathrm{L} 1$ & \multirow{4}{*}{$\mathrm{CE}$} & $0,37 * *$ & $0,73 * *$ & $0,96 * *$ & $0,20 *$ & $0,99 * *$ & $0,29 * *$ & $0,44 * *$ & $0,59 * *$ \\
\hline $\mathrm{L} 2$ & & $0,78 * *$ & $0,47 * *$ & $0,86 * *$ & $0,27 * *$ & $0,80 * *$ & $-0,04 \mathrm{~ns}$ & $0,37 *$ & $0,25 * *$ \\
\hline L3 & & $0,46^{* *}$ & $0,59 * *$ & $0,75^{* *}$ & $0,35^{* *}$ & $0,87 * *$ & $0,08 \mathrm{~ns}$ & $0,35^{*}$ & $0,54 * *$ \\
\hline L4 & & $0,51 * *$ & $0,81 * *$ & $0,90 * *$ & $0,24 * *$ & $0,88 * *$ & $0,14 \mathrm{~ns}$ & $0,38 *$ & $0,55^{* *}$ \\
\hline \multicolumn{10}{|c|}{ Período seco: diciembre-abril } \\
\hline $\mathrm{L} 1$ & \multirow{4}{*}{$\mathrm{CE}$} & $0,35^{* *}$ & $0,71 * *$ & $0,94 * *$ & $0,51 * *$ & $0,96 * *$ & $0,51 * *$ & $0,44 * *$ & $0,46 * *$ \\
\hline $\mathrm{L} 2$ & & $0,84 * *$ & $0,90 * *$ & $0,81 * *$ & $0,81 * *$ & $0,68 * *$ & $-0,09 \mathrm{~ns}$ & $-0,04 \mathrm{~ns}$ & $0,54 * *$ \\
\hline L3 & & $0,41 * *$ & $0,79 * *$ & $0,68 * *$ & 0,70 ** & $0,84 * *$ & $0,15 \mathrm{~ns}$ & $0,06 \mathrm{~ns}$ & $0,32 *$ \\
\hline L4 & & $0,41 * *$ & $0,88 * *$ & $0,84 * *$ & $0,51 * *$ & $0,89 * *$ & $0,27^{*}$ & $-0,13 \mathrm{~ns}$ & $0,50 * *$ \\
\hline
\end{tabular}

*significativo (5\%), **altamente significativo (1\%). 
iones no son suficientes o determinantes para estimar la respuesta de la CE, como para precisar la clasificación iónica de las aguas, sino que hay que considerar la interacción de un ion específico respecto de los demás que señalan Maia et al. (2001).

De igual forma, los niveles de la CE de las aguas freáticas en las diferentes localidades en los periodos lluviosos y secos pueden estar siendo influenciados por los ciclos marinos y deltaicos. Al respecto, Porta et al. (1999) y Jordan (2006) señalan que en los ciclos marinos, suelos cercanos a las zonas costeras presentan sales de origen marino y estas pueden provenir de capas freáticas subsuperficiales, aguas de mareas o de sales transportadas por el viento. Además, indican que la sal más frecuente en estas zonas es el cloruro de sodio, independientemente de la descarga de agua dulce aportada por el caudal fluvial de los ríos. Además, puede existir influencia del agua marina o de capas freáticas con alto grado de salinidad que ocasionan un desequilibrio de cationes y aniones por las variaciones de los volúmenes y concentraciones del agua dulce y de mar. Al respecto Sridhar et al. (2006) indican que la salinidad es el resultado de la temporada, porque en verano es alta y baja durante la estación de los monzones y los valores más altos registrados podrían ser atribuidos a la baja cantidad de precipitaciones, la mayor tasa de evaporación y debido también a la dominación del agua entre las épocas.

En el estuario del río Sinú bajo condiciones normales en la época seca se presentan las mareas más altas y la intrusión de aguas salobres al continente es mayor, pues se reducen los flujos hídricos fluviales. Esto ocasiona que se presenten los máximos valores de salinidad debido a la intensa evaporación, mientras que en época lluviosa se espera que presenten los valores mínimos en concentración de sales, debido al mayor aporte fluvial, causado por las precipitaciones y aumento en el caudal del río Sinú (CIOH, 1994; Sánchez et al., 1997). Sin embargo, los niveles de salinidad obtenidos para estos periodos pueden deberse a una posible influencia antrópica causada por la regulación de los niveles del río Sinú por parte de la hidroeléctrica Urra I, la que estaría explicando la tendencia de la salinidad de las aguas freáticas en el periodo lluvioso.

Otro proceso importante en la salinización de las aguas, es la denominada intrusión salina, que es el movimiento permanente o temporal del agua salada tierra adentro, desplazando al agua dulce.
$\mathrm{Al}$ respecto, Custodio y Llamas (1983) proponen las relaciones como $\mathrm{Cl} / \mathrm{HCO}_{3}$ y $\mathrm{SO}_{4} / \mathrm{Cl}$ (Xefteris et al. (2004) y la relación $(\mathrm{Ca}+\mathrm{Mg}) /(\mathrm{K}+\mathrm{Na})$ como indicadoras de la intrusión salina.

En la relación $\mathrm{Cl} / \mathrm{HCO}_{3}$ se indica que el cloruro prevalece en el agua de mar, y el bicarbonato caracteriza la mayor parte de las aguas como dulces, siendo muy baja su concentración en el agua de mar, por tanto, el rápido incremento de esta relación es un excelente trazador de la intrusión marina. Para esta relación se obtuvo que en el periodo lluvioso las localidades L1, L2, L3 y L4 presentaron valores de 4,57; 16,74; 18,26; 19,45 respectivamente, indicando que solamente en la localidad más alejada a la desembocadura (L1) presenta niveles característicos de agua dulce $(0,1$ a 5,0$)$ y el resto de localidades (L2 a L4) presentan valores próximos a los de aguas de mar (20 a 50). Para el periodo seco las localidades más próximas a la desembocadura del río Sinú (L3 y L4) presentaron valores $\mathrm{Cl} / \mathrm{HCO}_{3}$, típicos de aguas de mar $(21,7$ y 27,7$)$ y las localidades L1 y L2 mostraron valores de 2,7 y 15,6, los que corresponden a aguas dulces y transición entre aguas de río y de mar respectivamente.

En la relación $\mathrm{SO}_{4} / \mathrm{Cl}$, un descenso en los valores puede indicar que se ha producido una reducción de sulfatos, sin embargo, un aumento en los valores sería indicativo de una posible intrusión marina. Para este estudio, las aguas freáticas de las localidades presentan valores en esta relación entre 0,19 y 0,27 en el periodo lluvioso y entre 0,06 y 0,13 en el periodo seco, indicando una tendencia característica de aguas dulces en este periodo lluvioso y una posible influencia del proceso de intrusión salina en el periodo seco en la desembocadura del río Sinú. Explicado esto porque los valores se presentan dentro del rango 0,2 a 0,8 en aguas dulces y menores de 0,11 en aguas de mar según Custodio y Llamas (1983). De igual forma Xefteris et al. (2004) indican que una relación $(\mathrm{Ca}+\mathrm{Mg} /$ $(\mathrm{K}+\mathrm{Na})$ menor de 1 es una evidencia de intrusión salina, lo que corresponde con los valores obtenidos en este estudio, donde esta relación se encuentra entre 0,39 y 0,67 en el periodo lluvioso y entre 0,42 y 0,68 en el periodo seco.

Los resultados obtenidos en el estudio para las concentraciones de los iones $\mathrm{Ca}$ y $\mathrm{Mg}$ fueron muy similares en los dos periodos para todas las localidades. Esto puede deberse a procesos pedogenéticos que se presentan en la zona, los que 
en el transcurso del tiempo han generado suelos cuyo material predominante en la fracción fina es la montmorillonita, rica en minerales alcalinotérreos $\left(\mathrm{Ca}^{2+} \mathrm{y} \mathrm{Mg}^{2+}\right)$ (IGAC, 1983), lo que puede promover contenidos constantes de estos iones en las aguas freáticas.

La variabilidad de la clasificación de las aguas freáticas en el periodo seco respecto del lluvioso, posiblemente puede estar influenciada por una mayor participación de las interacciones de los iones de $\mathrm{Na}, \mathrm{Mg}$ y $\mathrm{K}$ con la $\mathrm{CE}$ en el periodo seco, lo que se refleja en la disminución de la magnitud de las correlaciones de $\mathrm{CE}$ con el ion $\mathrm{Cl}$ y $\mathrm{Na}$ y el aumento con los iones $\mathrm{K}$ y $\mathrm{Mg}$ principalmente. En el análisis general de la zona de estudio en el periodo seco, se determinaron aguas de tipo magnésicas, que de acuerdo con lo expresado anteriormente, es posible que se deba a que el ion $\mathrm{Mg}$ está presente en la composición de los materiales formadores de suelo de esta área, lo que estaría influenciando de forma importante en la composición iónica del agua freática. Al respecto, Maia et al. (2001) encontraron que el tipo de suelos presente en una región afecta en forma indirecta la calidad de las aguas y Santos et al. (1984) señalan que existe una relación entre la calidad del agua con el tipo de suelo.

\section{Conclusiones}

El agua freática presenta una salinización progresiva con la proximidad de las localidades a la desembocadura del río Sinú, asociada con las concentraciones de los iones salinizadores $\mathrm{Cl}, \mathrm{Na}$ y $\mathrm{Mg}$ con influencia en la $\mathrm{CE}$, asociada a ciclos marinos y deltaicos.

Relaciones iónicas como $(\mathrm{Ca}+\mathrm{Mg}) /(\mathrm{K}+\mathrm{Na})$, $\mathrm{Cl} / \mathrm{HCO}_{3}$ y $\mathrm{SO}_{4} / \mathrm{Cl}$ estarían evidenciando la influencia del proceso de intrusión salina en las aguas freáticas, especialmente durante el periodo seco.

Los modelos de regresión lineal múltiple obtenidos para las localidades del área de estudio presentan $R^{2} \geq 0,94$ indicando el alto grado de confiabilidad para estimar los valores de CE en función de los niveles de los iones que integran los modelos.

La clasificación de las aguas freáticas es afectada por los regímenes de precipitación, donde se evidencia el predominio de cloruros en el periodo lluvioso, mientras que en el periodo seco una disminución de estos cloruros estaría permitiendo una mayor interacción y participación de los iones de $\mathrm{Na}, \mathrm{Mg}$ y $\mathrm{K}$.

\section{Agradecimientos}

Al proyecto de investigación "Diagnóstico general del nivel de salinización en la zona aledaña a la desembocadura del río Sinú y su impacto socioeconómico: Período abril/2002noviembre/2008" y personal técnico del Laboratorio de Suelos y Aguas de la Universidad de Córdoba.

\section{Literatura Citada}

American Public Health Association (Apha). 1995. Standard methods for examination of water and wastewater. 19th Edition. Washington DC. 380 pp.

Ayers, R.; Westcot, D.

1985.Water quality for agriculture. Food and Agriculture Organization. Rome, Italy.

Bustamante, $\mathrm{M}$.

1993. Drenaje de tierras agrícolas y sus aplicaciones. Universidad Nacional de Colombia, Sede Medellín-Colombia. 247 pp. Cortés-Jiménez, J.; Troyo-Diéguez, E.; Murillo-Amador, B.;

García-Hernández, J.; Garatuza-Payán.; Sang, L. 2009. Índices de calidad del agua del acuífero del valle del Yaqui, Sonora. Terra Latinoamericana, 27 (2): 133-141.

Coto, B.; Martos, C.; Peña, J.L.; Rodríguez, R. y Pastor, G. 2012. Effects in the solubility of $\mathrm{CaCO}_{3}$ : Experimental study and model description. Fluid Phase Equilibria, 324: 1-7. Custodio, E.; Llamas, M.

1983. Hidrología subterránea. Tomo II. Segunda edición. Omega, Barcelona, España. 2350 pp.
CIOH-Centro de Investigaciones Oceanográficas e Hidrológicas. 1994. Estudio oceanográfico de recuperación de playas sector La Perdiz Puerto Viejo. Golfo de Morrosquillo. Cartagena de Indias D.T. y C. 189 pp.

Ferreira, P.

1997. Aspectos físico-químicos do solo, In: Gheyi, H.R.; Queiroz, J.E.; Medeiros, J.F. de. (ed). Manejo e controle da salinidade na agricultura irrigada. Campina Grande: UFPB, pp. 37-67.

Holanda, J.; Amorim, J.

1997. Qualidade da água para irrigação, In: Gheyi, H.R.; Queiroz, J.E.; Medeiros, J.F. de.(ed). Manejo e controle da salinidade na agricultura irrigada. Campina Grande: UFPB, pp. 137-169.

IGAC.

1983. Instituto Geográfico “Agustín Codazzi”, Subdirección Agrológica. Estudio General de los suelos de los municipios que conforman la parte media y baja de la cuenca del Sinú (Departamento de Córdoba). República de Colombia, 
Ministerio de Hacienda y Crédito Público. Bogotá, D.E. $236 \mathrm{pp}$.

Jordan, A.

2006. Manual de edafología. Departamento de Cristalografía, Mineralogía y Química Agrícola de la Universidad de Sevilla. Disponible en: URL:libnet.unse.edu.ar/1bi/ba/ cefaya/cdig/000005.pdf. Consultado: [12-Agosto-2012].

Maia, C.E.; Morais, E.R.C. de; Oliveira, M.

2001. Classificação da composição iônica da agua de irrigação usando regressão linear múltipla. Revista Brasileira de Engenharia Agrícola e Ambiental, 5 (1): 55-59.

Negrete, F.; Tamara, R.; Camargo, L.; Arguelles, J.

2011. Manejo de la profundidad del nivel freático en el suelo para mejorar la producción de los cultivos de maíz y algodón en la microrregión Valle del Sinú. Boletín técnico Corpoica, $20 \mathrm{pp}$.

Palencia, G.; Mercado, T.; Combatt, E.

2006. Estudio agroclimático del departamento de Córdoba. Facultad de Ciencias Agrícolas, Universidad de Córdoba. 126 pp.

Porta, J.; López-Acevedo, M.; Roquero, C.

1999. Edafología para la agricultura y el medio ambiente. Editorial Mundi-prensa, Madrid. $2^{\text {a }}$ edición, 849 pp.

Rodríguez-Pérez, L.

2006. Implicaciones fisiológicas de la osmorregulación en plantas. Agronomía Colombiana, 24 (1): 28-37.
Sánchez, H.; Álvarez, R.; Pinto, F.; Sánchez, A.; Pino, J.; García, I.; Acosta, M.

1997. Diagnóstico y zonificación preliminar de los manglares del Caribe de Colombia. Proy. PD 171/91 Rev. 2 (F) Fase I. Conservación y manejo para el uso múltiple y el desarrollo de los manglares de Colombia, MADT/OIMT. Santa fe de Bogotá D.C., Colombia. 511 pp.

Santos, J.; Azevedo, S.; Mistretta, G.

1984. Novos aspectos da salinização das aguas subterrâneas do cristalino do Rio Grande do Norte, São Paulo, SP: IPT, 27 pp.

Shalhevet, J.; Kamburov, J.

1976. Irrigation and salinity: A world-wide survey. New Delhi: CaxtonPress. 106 pp.

Silva, F.

2000. Fundamentos para la interpretación de análisis de suelo, plantas y aguas para riego. Tercera edición. SCCS: Bogotá. $323 \mathrm{pp}$.

Sridhar, R.; Thangaradjou, T.; Senthil, K.; Kannan, L.

2006. Water quality and phytoplankton characteristics in the Palk Bay, Southeast Coast of India J. environ. Biol., 27: 561-566.

Tanji, K.

1990. Agricultural salinity assessment and management. Published by ASCE: New York. 619 pp.

Xefteris, A.; Anastasiadis, P.; L. Atinopoulus, P.

2004. Groundwater chemical characteristics in Kalamaria plain, Halkidiki peninsula, Grecce. FreseniusEnviron. Bull, 13: 1159-1167. 
